\title{
CELL-LIKE DECOMPOSITIONS OF HOMOGENEOUS CONTINUA
}

\author{
JAMES T. ROGERS, JR. ${ }^{1}$
}

\begin{abstract}
Certain decompositions of homogeneous continua are shown to be cell-like. In particular, the aposyndetic decomposition described by F. B. Jones of a homogeneous, decomposable continuum is cell-like, and we prove that any homogeneous decomposable continuum admits a continuous decomposition into mutually homeomorphic, indecomposable, homogeneous, cell-like terminal continua 80 that the quotient space is an aposyndetic homogeneous continuum.
\end{abstract}

D. C. Wilson [8] has shown that a monotone, completely regular map $f: X \rightarrow Z$ of the $n$-dimensional continuum $X$ onto the nondegenerate continuum $Y$ has the property that $H^{n}\left(f^{-1}(z)\right)=0$, for all $z$ in $Z$. In particular, if $n=1$, then the point inverses of $f$ are acyclic continua.

More recently, Mason and Wilson [5] have shown that if $n=1$, then the point inverses of $f$ are tree-like continua, that is, $f$ is a cell-like map.

Since the projection maps of a product space onto the factors are completely regular maps, one cannot, in general, extend the Mason-Wilson result to higher dimensions.

The impetus for the Mason-Wilson result, however, was the author's applications $[6,7]$ of completely regular maps to certain monotone decompositions of homogeneous continua. In this paper, we show that the completely regular, monotone maps arising as quotient maps of these decompositions are cell-like maps. In particular, Jones' aposyndetic decomposition of a homogeneous, decomposable continuum is a decomposition of that continuum into cell-like sets.

A continuum $X$ is cell-like if each mapping of $X$ into a compact ANR is inessential. If the map $f$ is inessential, we write $f \simeq 0$. A continuum is cell-like if and only if it has trivial shape.

A map is cell-like if each of its point inverses is cell-like.

The following theorem is classical.

Wirecutting TheOREM. Let $A$ and $B$ be closed subsets of the compact space $M$. If no connected subset of $M$ intersects both $A$ and $B$, then there exist disjoint closed subsets $M_{1}$ and $M_{2}$ of $M$ such that $A \subset M_{1}, B \subset M_{2}$, and $M=M_{1} \cup M_{2}$.

A subcontinuum $Z$ of the continuum $X$ is said to be terminal if each subcontinuum $Y$ of $X$ such that $Y \cap Z \neq \varnothing$ satisfies either $Y \ddot{\subset} Z$ or $Z \subset Y$.

The proof of the next theorem is similar to that of [2, Theorem 2].

Received by the editors September 11, 1981.

1980 Mathematics Subject Classification. Primary 54F20, 54F50.

Key words and phrases. Cell-like decomposition, aposyndetic, homogeneous, completely regular map.

${ }^{1}$ This research was partially supported by NSF grant number MCS- 8101565 .

(c) 1983 American Mathematical Society 0002-9939/82/0000-0388/\$02.00 
THEOREM 1. If $A$ is a terminal subcontinuum of the continuum $X$, if $B$ is a subcontinuum of $X$ disjoint from $A$, and if $f: A \rightarrow Y$ is a map of $A$ into the $A N R$ $Y$, then there exists a map $F: X \rightarrow Y$ such that $F \mid A=f$ and $F \mid B \simeq 0$.

Proof. There exists an open set $U$ in $X-B$ containing $A$ and a map $g: U \rightarrow$ $Y$ extending $f$. There exists an open neighborhood $V$ of a point $a$ in $A$ such that $V \subset U$ and $g \mid V \simeq 0$, since $Y$ is locally contractible. Since $A-V$ and $X-U$ are closed subsets of $X-V$ such that no connected subset of $X-V$ meets both $A-V$ and $X-U$, the Wirecutting Theorem implies that $X-V$ is the union of the disjoint closed sets $X_{1}$ and $X_{2}$, with $A-V \subset X_{1}$ and $X-U \subset X_{2}$.

Thus $X_{1} \cup A$ and $X_{2}$ are disjoint closed subsets of $X$. Let $h: X \rightarrow I$ be a Urysohn map with $h\left(X_{1} \cup A\right)=0$ and $h\left(X_{2}\right)=1$. Let $M=h^{-1}\left(\left[0, \frac{1}{2}\right]\right)$ and $N=h^{-1}\left(\left[\frac{1}{2}, 1\right]\right)$. Then $X=M \cup N, A \subset M, X-U \subset N$, and $M \cap N \subset V-A$. Since the map $g \mid M \cap N$ is homotopic to a constant map, there exists an inessential map $k: N \rightarrow Y$ such that $k|M \cap N=g| M \cap N$.

The map $F: X \rightarrow Y$ defined by

$$
F(x)= \begin{cases}g(x), & x \in M \\ k(x), & x \in N\end{cases}
$$

is the desired extension of $f$.

A map $g: X \rightarrow Z$ between continua is completely regular if for each $\delta>0$ and each point $z$ in $Z$, there exists an open set $V$ in $Z$ containing $z$ such that if $z^{\prime} \in V$ then there is a homeomorphism $h$ from $g^{-1}(z)$ to $g^{-1}\left(z^{\prime}\right)$ such that $d(x, h(x))<\delta$, for each $x$ in $g^{-1}(z)$. Each completely regular map is open.

THEOREM 2. Let $g: X \rightarrow Z$ be a monotone, completely regular map of the continuum $X$ onto the nondegenerate continuum $Z$. Let $z_{1}$ be a point of $Z$. If $g^{-1}\left(z_{1}\right)$ is a terminal subcontinuum of $X$, then $g$ is a cell-like map.

PROOF. Let $Y$ be a compact ANR, and let $f: g^{-1}\left(z_{1}\right) \rightarrow Y$ be a map. Let $z_{2}$ be another point of $Z$, and let $F: X \rightarrow Y$ be an extension of $f$ such that $F \mid g^{-1}\left(z_{2}\right) \simeq 0$.

Since $Y$ is a compact ANR, there exists $\epsilon>0$ such that for any space $W$ and for any two maps $\alpha, \beta: W \rightarrow Y, d(\alpha, \beta)<\epsilon$ implies $\alpha \simeq \beta$. Let $\delta$ be a positive number such that $d\left(x, x^{\prime}\right)<\delta$ implies $d\left(F(x), F\left(x^{\prime}\right)\right)<\epsilon$.

Let $Z_{2}=\left\{z \in Z: F \mid g^{-1}(z) \simeq 0\right\}$. We show that $Z_{2}$ is open. Let $z \in Z_{2}$. Since $g$ is completely regular, these exists an open set $V$ in $Z$ containing $z$ such that if $z^{\prime} \in V$, then there is a homeomorphism $h$ from $g^{-1}(z)$ to $g^{-1}\left(z^{\prime}\right)$ such that $d(x, h(x))<\delta$, for each $x$ in $g^{-1}(z)$. Hence $d\left(F\left|g^{-1}\left(z^{\prime}\right), F\right| g^{-1}(z) \circ h^{-1}\right)<\epsilon$, and so $F \mid g^{-1}\left(z^{\prime}\right) \simeq$ $F \mid g^{-1}(z) \circ h^{-1}$. But the latter map is inessential, since $z \in Z_{2}$, and so $z^{\prime} \in Z_{2}$. Hence $Z_{2}$ is open. A similar proof shows that $Z_{2}$ is closed.

Since $Z_{2}$ is a nonempty open and closed subset of the connected set $Z$, it follows that $Z_{2}=Z$ and hence $f \simeq 0$. Thus each mapping of $g^{-1}\left(z_{1}\right)$ into a compact ANR is inessential, and hence $g^{-1}\left(z_{1}\right)$ is cell-like. Since point inverses of completely regular maps defined on continua are homeomorphic, this implies that $g$ is a cell-like map.

The homeomorphism group $H$ of a continuum $X$ is said to respect the decomposition $\mathcal{G}$ of $X$ if $G \in \mathcal{G}$ implies $h(G) \in \mathcal{G}$, for each homeomorphism $h$ in $H$.

The following theorem is due to E. Dyer (see [3] for a simple proof).

THEOREM 3. Let $X$ and $Y$ be nondegenerate metric continua and let $f: X \rightarrow Y$ be a monotone open surjection. Then there exists a dense $G_{\delta}$-subset $A$ of $Y$ having 
the following property: for each $y \in A$, for each continuum $B \subset f^{-1}(y)$, for each $x$ from the interior of $B$ in $f^{-1}(y)$ and for each neighborhood $U$ of $B$ in $X$, there exists a continuum $Z \subset X$ containing $B$ and a neighborhood $V$ of $y$ in $Y$ such that $x \in Z^{0}$, $(f \mid Z)^{-1}(V) \subset U$ and $f \mid Z: Z \rightarrow Y$ is a monotone surjection.

THEOREM 4. Let $X$ be a homogeneous continuum. Let $\mathcal{G}$ be a partition of $X$ into terminal subcontinua such that the homeomorphism group of $X$ respects $\mathcal{G}$. Then

(1) the partition $\mathcal{G}$ is a continuous decomposition of $X$.

(2) The quotient map $\pi: X \rightarrow Z$ of $X$ onto the quotient space $Z$ is completely regular.

(3) $Z$ is a homogeneous continuum.

(4) The elements of $\mathcal{G}$ are mutually homeomorphic, indecomposable, homogeneous, cell-like continua.

ProOF. Parts (1), (2), and (3) have been proved in [7, Theorem 4]. Since the homeomorphism group of $X$ respects $\mathcal{G}$, it follows that the elements of $\mathcal{G}$ are mutually homeomorphic and homogeneous. Theorem 2 implies that the elements of $\mathcal{G}$ are cell-like. As in [4], Dyer's Theorem and the fact that the elements of $\mathcal{G}$ are terminal continua imply that the proper subcontinua of one (and hence all) of the elements of $\mathcal{G}$ have empty interiors in that element. Hence the elements of $\mathcal{G}$ are indecomposable.

An important application of this theorem is the following improvement of Jones' Aposyndetic Decomposition Theorem [1]. In the case that $X$ is a curve, this improvement is already known from results of Rogers [6] and Mason and Wilson $[\mathbf{5}]$.

THEOREM 5. Suppose $X$ is a homogeneous, decomposable continuum. Then $X$ admits a continuous decomposition into mutually homeomorphic, indecomposable, homogeneous, cell-like terminal continua so that the quotient space is an aposyndetic homogeneous continuum.

ProOF. Jones [1] has shown that his decomposition of $X$ consists of terminal continua. Furthermore, the homeomorphism group of $X$ respects this decomposition.

Question 1. Is each homogeneous, indecomposable, cell-like continuum tree-like? Question 2. Can the aposyndetic decomposition of Theorem 5 raise dimension?

\section{REFERENCES}

1. F. B. Jones, On a certain type of homogeneous plane continuum, Proc. Amer. Math. Soc. 6 (1955), 735-740.

2. J. Krasinkiewicz, Mapping properties of hereditarily indecomposable continua, preprint.

3. - On two theorems of Dyer, preprint.

4. T. Maćkowiak and E. D. Tymchatyn, Continuous mappings on continua. II, preprint.

5. A. Mason and D. C. Wilson, Monotone mappings on $n$-dimensional continua, preprint.

6. J. T. Rogers, Jr., Completely regular mappings and homogeneous, aposyndetic continua, Canad. J. Math. 33 (1981), 450-453.

7. - Decompositions of homogeneous continua, Pacific J. Math. 99 (1982), 137-144.

8. D. C. Wilson, Completely regular mappings and dimension, Bull. Amer. Math. Soc. 76 (1970), 1057-1061.

Department of Mathematics, Tulane University, New Orleans, Louisiana 